\title{
Inhibition of monocyte chemoattractant peptide-1 decreases secondary spinal cord injury
}

\author{
XUESONG ZHANG ${ }^{1}$, ${\text { CHAO } \mathrm{CHEN}^{1} \text {, SHENGZHONG MA }{ }^{2} \text {, YAN WANG }{ }^{1}, \text { XUELIAN ZHANG }^{3} \text { and XIAOJING SU }}^{1}$ \\ ${ }^{1}$ Spine Department, General Hospital of PLA, Beijing 100853; ${ }^{2}$ Spine Department, \\ The Second Hospital of Shandong University, Jinan, Shandong 250033; ${ }^{3}$ Endocrine Department, \\ China-Japan Friendship Hospital, Beijing 100029, P.R. China
}

Received January 9, 2013; Accepted May 13, 2013

DOI: $10.3892 / \mathrm{mmr} .2015 .3330$

\begin{abstract}
Previous studies have suggested that impairment secondary to mechanical injury is a major cause of irreversible damage to the spinal cord. Inflammatory chemokines have been shown to play an important role in the pathological and physiological consequences of secondary spinal cord injury (SCI). The aim of the present study was to evaluate how changes in the expression levels of the cellular chemokine, monocyte chemoattractant peptide-1 (MCP-1), and the chemotaxis of inflammatory cells (monocytes and macrophages) are involved in the process of SCI. RNA interference methods were used to study the mechanisms that protect residual neurons after SCI in an attempt to explore novel, early interventions for managing SCI. Our results suggested that inhibiting inflammation alleviates nerve cell injury caused by apoptosis and provides a potentially important approach for the future treatment of secondary SCI.
\end{abstract}

\section{Introduction}

Spinal cord injury (SCI) is a serious complication of vertebral fractures. Primary SCI, caused by vertebral fracture, results in an irreversible mechanical injury to the spinal cord (1). Secondary SCI is often a sequel to the primary injury that results from arterial disruption, electrolyte imbalance, thrombosis or hypoperfusion due to shock. Secondary SCI is associated with complex pathological and physiological processes that have widespread consequences. For this reason, secondary SCI may be more harmful to the nervous system than the primary injury.

Secondary SCI is both preventable and reversible. Physical therapy and the early application of drug therapy can have a significant impact on patient prognosis. However, to date,

Correspondence to: Dr Xuelian Zhang, Endocrine Department, China-Japan Friendship Hospital, 2 Yinghua East Road, Chaoyang, Beijing 100029, P.R. China

E-mail: zhangxuelian666@163.com

Key words: secondary spinal cord injury, monocyte chemoattractant peptide-1, RNA interference, inflammation, apoptosis therapeutic options for secondary SCI management are limited and the development of clinical and therapeutic modalities based on new technology is the subject of ongoing research (2).

The roles of apoptosis and necrosis in secondary SCI have been reported previously (2). Neuronal and glial cell death does not appear to be due to direct damage. Studies using animal models and human tissue samples have revealed that cell damage is associated with the apoptosis of neuronal and glial cells, and that these processes may be key factors in secondary SCI $(3,4)$.

The extent of apoptosis and cavity formation in the spinal cord after secondary SCI are important factors that require further investigation. These changes have been shown to decrease when hemorrhaging is reduced by drug treatment (5). Other research has demonstrated that inflammation occurs following secondary SCI, leading to the abnormal apoptosis of neuronal and glial cells, thereby exacerbating the situation.

Increases in the mRNA levels of the cytokine factor monocyte chemoattractant peptide-1 (MCP-1) have been observed following the occurrence of secondary SCI (6). This finding may shed light on the mechanism that precipitates tissue inflammation (7).

In the present study, an astrocyte cell line from rat brain tissue and a rat model of Nyström's posterior spinal cord compression injury were used to explore the association between MCP-1 expression and secondary SCI.

\section{Materials and methods}

Cell culture. Whole brains harvested from newborn rats under sterile conditions were placed in D-Hank's solution containing $10 \%$ fetal bovine serum and 5\% DMEM-F12 (Gibco, Carlsbad, CA, USA). The pallium was separated under a dissecting microscope and the resulting tissue was exposed to trypsin. Cells were collected by centrifugation and plated onto Petri dishes at a density of $1.5 \times 10^{7}$ cells per dish. The cells were cultured for 10 days at $37^{\circ} \mathrm{C}$ in the presence of $5 \% \mathrm{CO}_{2}$ and $95 \% \mathrm{O}_{2}$. High purity astrocytes were prepared following separation, purification and subculture.

Animal models. Healthy, closed population, male Sprague-Dawley (SD) rats weighing 260-300 g were used for the animal models. The rats were given free access to food and water in natural light at room temperature. Improved 
Nyström surgery was performed in order to produce the spinal cord injury by applying compression for $5 \mathrm{~min}$ with a $35-\mathrm{g}$ weight. Following surgery, the rats were raised separately at room temperature. In the control group, the lamina of the vertebral arch was removed to expose the dura mater. A total of $80 \mathrm{SD}$ rats were used to establish the animal model. In total, 20 rats died; four during the breeding period, six during anesthesia for surgery and ten during the observation period once the model had been established. The 60 surviving rats were randomly divided into five groups, with 12 rats in each group; group 1 (untreated group), group 2 (sham surgery group), group 3 (rat spinal cord compressed for $1 \mathrm{~min}$ ), group 4 (rat spinal cord compressed for $5 \mathrm{~min}$ ) and group 5 (rat spinal cord compressed for $10 \mathrm{~min}$ ). All animal studies were approved by the Institutional Animal Care and Use Committee of the Second Military Medical University (Shanghai, China) and all experimental procedures were carried out in accordance with institutional guidelines.

RNA manipulation studies. A small interfering RNA expression vector, pSilencer 3.1-H1 puro (Ambion, Foster City, CA, USA) containing MCP-1 and MCP-1 $\beta$ targeting sequences, and appropriate scrambled controls were constructed according to the manufacturer's instructions. In order to select the optimum targeting sequences, the MCP-1 coding sequences were submitted to the Ambion siRNA target finder website (http://www.ambion.de/en/) for prediction. The following two small interfering (siRNA) sequences and a scramble siRNA, as a negative siRNA control, were used for the RNA interference experiments: MCP-1, 5'-GAT CCC GTGCCCCACTCACCT GCTGCT TCA AGA GAG CAGC AGG TGA GTG GGG CAC TTT TTT GGA AA-3' and 5'-AGC TTT TCC AAA AAA GTG CCC CAC TCA CCT GCTG CTC TCT TGA AGC AGC AGGTGAGTGGGGCACGG-3'; and scramble siRNA, 5'-ATGGACAGAATAAATGGACTT-3'. Constructed pSilencer-MCP-1 and pSilencer-MCP-1 $\beta$ were transfected into the astrocyte cell line using Lipofectin (Invitrogen, Carlsbad, CA, USA).

Protein and $m R N A$ quantification. Changes in the expression of MCP-1 and MCP-1 $\beta$ were confirmed by the measurement of mRNA and protein levels. Total RNA was extracted from cell line samples using TRIzol reagent (Invitrogen) according to the manufacturer's instructions. Reverse transcription polymerase chain reaction (RT-PCR) was used to evaluate the mRNA expression levels. The primers used were as follows (100 $\mathrm{nM}$ each): MIP-1 $\beta$ upstream, 5'-TCTGCGATTCAGTGCTGTCAGC-3' and downstream, 5'-GATTTGCCTGCCTTTTTTGGTC-3'; MCP-1 upstream, 5'-CCTGTTGTTCACAGTTGCTGCC-3' and downstream, 5'-TCTACAGAAGTGCTTGAGGTGGTTG-3'; and $\beta$-actin upstream, 5'-ATGGTGGGTATGGGTCAGAA-3' and downstream, 5'-GCTGTGGTGGTGAAGCTGTA-3'. Cell extracts were prepared in NP-40 lysis buffer $(0.1 \%$ NP-40, $150 \mathrm{mM} \mathrm{NaCl}, 1 \mathrm{mM}$ EDTA, $50 \mathrm{mM}$ Tris $\mathrm{pH}$ 8.0, $1 \mathrm{mM}$ sodium vanadate, $1 \mathrm{mM}$ phenylmethylsulfonyl fluoride) supplemented with a protease inhibitor cocktail (Sigma, St. Louis, MO, USA; P-8340). Protein concentrations were determined using a Bradford assay (Bio-Rad, Hercules, CA, USA). Equivalent amounts of protein (10-30 $\mu \mathrm{g})$ were electrophoretically resolved using SDS-PAGE on 6-10\% gels. MCP-1 and MCP-1 $\beta$ primary antibodies were used for western blotting to detect the protein expression levels.

Immunohistochemistry. The astrocyte cell line and rat tissues were subjected to immunohistochemistry. Paraffin-embedded sections were cleared and incubated at $37^{\circ} \mathrm{C}$ for $10 \mathrm{~min}$ with $0.1 \%$ Pronase (Roche, Mannheim, Germany; \#165 921) in $0.1 \%$ $\mathrm{CaCl}_{2}$ at $\mathrm{pH}$ 7.8. The suspension was blocked by exposure to $3 \%$ hydrogen peroxide in TBST for $10 \mathrm{~min}$. The tissues were further washed and blocked with a biotin blocking system (Dako, Carpinteria, CA, USA; X0590), followed by washing and exposure to $10 \%$ normal rabbit serum for $10 \mathrm{~min}$ at room temperature. The cells were then incubated for $1 \mathrm{~h}$ at room temperature with polyclonal rabbit anti-mouse MCP-1 antibody (1:800 diluted; Santa Cruz Biotechnology, Inc., Dallas, TX, USA). This was followed by incubation for $30 \mathrm{~min}$ at room temperature with biotinylated rabbit anti-mouse serum (1:100 diluted; Dako Denmark A/S, Glostrup, Denmark) and finally by incubation for $30 \mathrm{~min}$ at room temperature with Strep-ABC complex (1:100 diluted; Dako Denmark A/S). The sections were developed for $20 \mathrm{~min}$ at room temperature using an AEC substrate kit (Vector Lab, Burlingame, CA, USA; SK-4200), counterstained with hematoxylin and, following drying, were mounted with Dako aqueous mount (Dako Denmark A/S).

Terminal deoxynucleotidyl transferase dUTP nick end labeling (TUNEL) assay. TUNEL is a common method for detecting the DNA fragmentation that results from apoptotic signaling cascades. The technique was applied to an astrocyte cell smear and tissue samples using an in situ cell death detection kit (Roche). Cell smear preparation, tissue sample treatment and TUNEL analysis were performed according to the manufacturer's instructions. Cells were mounted under a glass coverslip and analyzed with a fluorescence microscope to observe apoptosis.

\section{Results and Discussion}

RNA inhibition (RNAi) plasmid inhibits MCP-1 expression. MCP-1 plays an important role in the inflammatory response induced by secondary SCI $(6,8)$. RNAi decreased the expression of MCP-1 in animal models and astrocyte cell lines (Figs. 1 and 2). This finding provides a basis for further studies investigating the effect of MCP-1 expression on SCI.

Inflammatory response after secondary SCI. Astrocytes are a major cell type found in the cerebrum. These cells constitute an inner microenvironment essential for neuron development. They also contain a number of neurotransmitters, which facilitate neuronal conduction. It has been reported that astrocytes in the region of the blood-brain (spinal cord) barrier are involved in inflammatory immune responses in the brain and spinal cord. These cells excrete inflammatory chemokines involved in the recruitment of peripheral blood monocytes, macrophages and T lymphocytes (9-11).

It has previously been demonstrated that an inflammatory response occurs during the first few hours after SCI. This involves activation of the remaining astrocytes and the infiltration of peripheral inflammatory cells; the majority of 
Table I. Positive cells at various time-points after spinal cord injury, as determined by a TUNEL assay.

Number of positive cells (mean \pm SD)

\begin{tabular}{|c|c|c|c|c|}
\hline \multirow[b]{3}{*}{ Time after spinal cord injury } & \multirow{2}{*}{\multicolumn{2}{|c|}{ Grey matter }} & & \\
\hline & & & \multicolumn{2}{|c|}{ White matter } \\
\hline & RNAi & Control & RNAi & Control \\
\hline $1 \mathrm{~h}$ & $0 \pm 1$ & $0 \pm 1$ & $0 \pm 1$ & $0 \pm 1$ \\
\hline $8 \mathrm{~h}$ & $40 \pm 10$ & $42 \pm 8^{\mathrm{a}}$ & $7 \pm 2$ & $9 \pm 1$ \\
\hline $24 \mathrm{~h}$ & $10 \pm 3$ & $95 \pm 12^{\mathrm{a}}$ & $6 \pm 1$ & $35 \pm 5^{\mathrm{a}}$ \\
\hline $72 \mathrm{~h}$ & $5 \pm 3$ & $50 \pm 6^{\mathrm{a}}$ & $9 \pm 4$ & $413 \pm 10^{\mathrm{a}}$ \\
\hline
\end{tabular}

${ }^{a} \mathrm{P}<0.05$ vs. $1 \mathrm{~h}$ after spinal cord injury. TUNEL, terminal deoxynucleotidyl transferase dUTP nick end labeling; RNAi, RNA inhibition.

these are neutrophils, granulocytes, monocytes and macrophages (12). Glial cells and vascular endothelial cells in the central nervous system produce inflammatory cytokines (13).

Changes that occurred at different stages of injury in the rat SCI model are shown in Fig. 3. Sections from the animal model revealed that the most severe SCI occurred after $24 \mathrm{~h}$, where the necrosis of glial cells, a reduction in the number of neurons and disorder of white matter structure with only a few nerve cells remaining around the area of damage were observed. Injured neurons were detected, mainly around small vessels.

Following 2 weeks of SCI, glial fibers gradually appeared in the grey matter, accompanied by small cystic degeneration. The central canal began to reconstruct, changes in white matter structure were observed and the abundant proliferation of glial cells was detected.

Inhibiting $\mathrm{MCP}$-1 reduces inflammatory changes. Inflammatory cytokines, including IL-1 $\beta$ and TNF- $\alpha$, are hypothesized to cause the accumulation and increased expression of MCP-1 in rat astrocytes, and these changes may be involved in important signaling pathways (14). In the same experiments, abnormally high expression levels of these cytokines were shown to be correlated with the development of inflammation.

In rat models of cerebral cortical nitrocellulose membrane needle-stick injury, elevated MCP-1 expression has been linked to astrocyte activation and the infiltration of monocytes and macrophages (15). It has been demonstrated that mechanical injury to the central nervous system causes astrocytes to produce MCP-1 (11).

The expression of inflammatory chemokines MCP-1 and MIP-1 $\beta$ were significantly increased in the secondary SCI rat model (Fig. 4), which is consistent with previous studies $(6,16)$.

In this study, MCP-1 and MIP-1 $\beta$ expression increased after SCI and monocyte and macrophage infiltration also increased in the damaged areas. After antisense gene intervention, the expression levels of MCP-1 and MIP- $1 \beta$ were reduced and the infiltration of monocytes and macrophages decreased.

The expression of TNF- $\alpha$ in response to spinal injury decreased after RNAi inhibited the expression of MCP-1 (Fig. 5), demonstrating that the inflammatory response induced by $\mathrm{SCI}$ is associated with MCP-1 expression.

The observation of rat tissue sections revealed that significant changes occurred in the activity of monocytes and macrophages following the inhibition of MCP-1 expression.

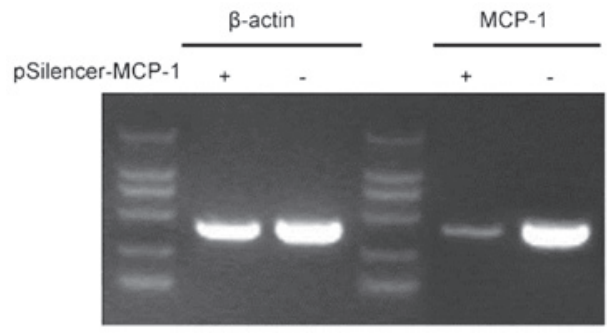

Figure 1. RNAi plasmid inhibits MCP-1 expression in astrocyte cell lines MCP-1, monocyte chemoattractant peptide-1; RNAi, RNA inhibition.

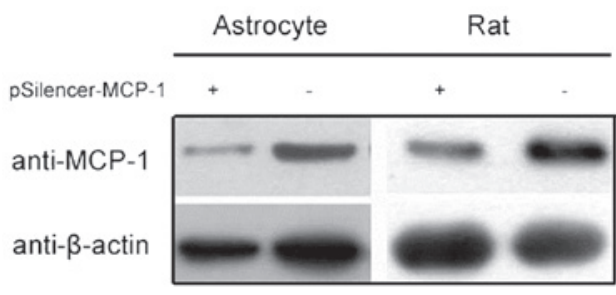

Figure 2. MCP-1 protein expression is inhibited by RNAi plasmid in astrocyte cell lines and in the rat spinal cord injury model. MCP-1, monocyte chemoattractant peptide-1; RNAi, RNA inhibition.

Reduction in MCP-1 expression inhibits neural cell apoptosis induced by secondary SCI. Following the emergence of secondary SCI, apoptosis induced by a series of inflammatory responses following the primary SCI resulted in neural cell necrosis. We demonstrated that the number of TUNEL-positive cells reached a peak at $24 \mathrm{~h}$ after the primary SCI (Table I). The TUNEL-positive cells were mainly glial cells and were predominantly distributed in grey matter. The number of TUNEL-positive glial cells in the white matter reached a peak $\sim 72 \mathrm{~h}$ later. However, in the RNAi group, the number of TUNEL-positive cells in the grey and white matter were lower than the control group following inhibition of MCP-1 expression by RNAi (Table I).

Apoptosis in secondary SCI is a process in which numerous regulatory factors are involved, with the caspase family as the core component. Low caspase-3 expression is usually detected in the spinal cord nerve cells of normal rats; however, its expression has been found to increase several 
A

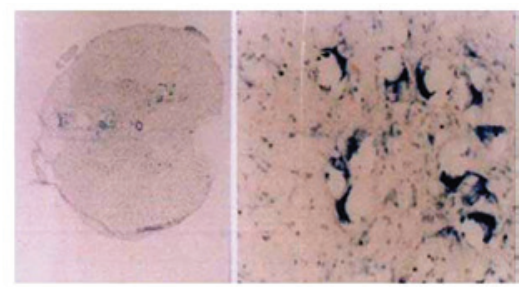

B

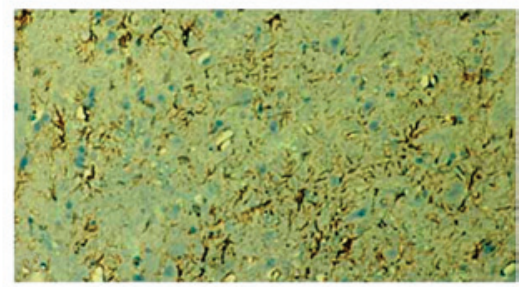

C

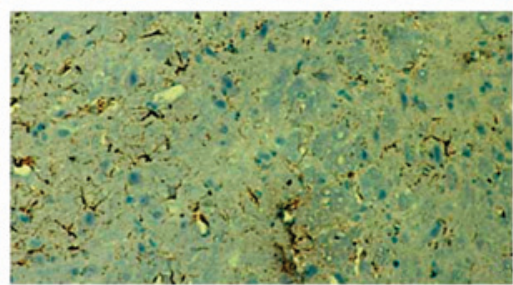

D

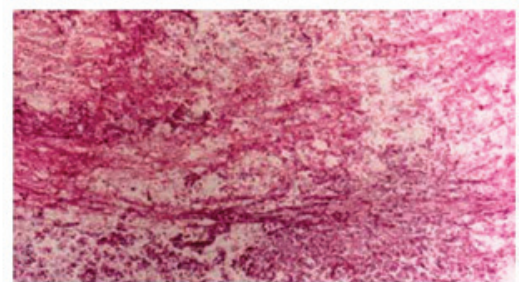

$\mathbf{E}$

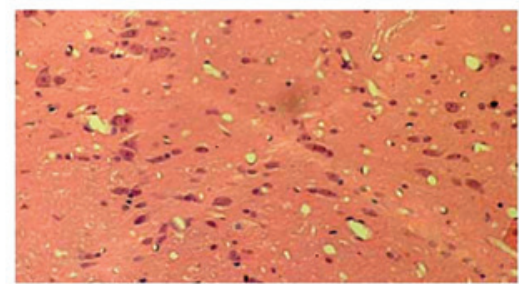

$\mathbf{F}$

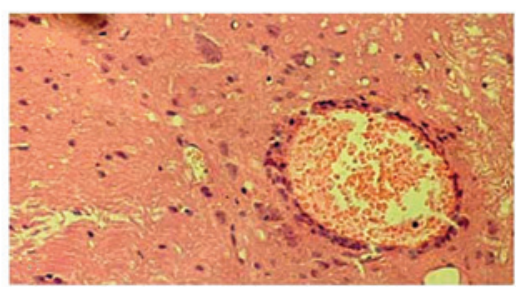

Figure 3. Changes at different stages of injury in the rat spinal cord injury (SCI) model. (A) Nissl staining of the damaged area 2 weeks after severe SCI (longitudinal section; magnification, x200). (B) Glial fibrillary acidic protein (GFAP) staining of the damaged area 1 week after mild SCI (longitudinal section; magnification, x200). (C) GFAP staining of the damaged area $24 \mathrm{~h}$ after moderate SCI (longitudinal section; magnification, x200); (D) Hematoxylin and eosin (H\&E) staining of the damaged area 2 weeks after severe SCI (longitudinal section; magnification, x200). (E) H\&E staining of the damaged area $4 \mathrm{~h}$ after mild SCI (longitudinal section; magnification, x200). (F) H\&E staining of the damaged area $24 \mathrm{~h}$ after moderate SCI (transverse section; magnification, $\mathrm{x} 200$ ).

hours after SCI, reaching a peak after 2-3 days (17). This time profile is consistent with the apoptotic process. Furthermore, it has been observed that caspase-1 activity is inhibited after the emergence of SCI in mice lacking dynorphin, suggesting that caspase-1 protects the nervous tissues following the occurrence of SCI (18).
A

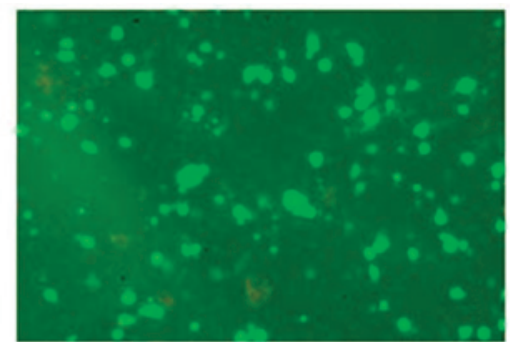

B

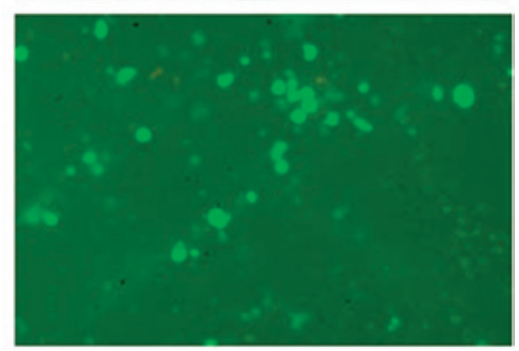

Figure 4. Change in MCP-1 expression prior to and following the emergence of spinal cord injury in rat models. (A) $24 \mathrm{~h}$ after the emergence of spinal cord injury; (B) prior to the emergence of spinal cord injury. MCP-1, monocyte chemoattractant peptide-1.

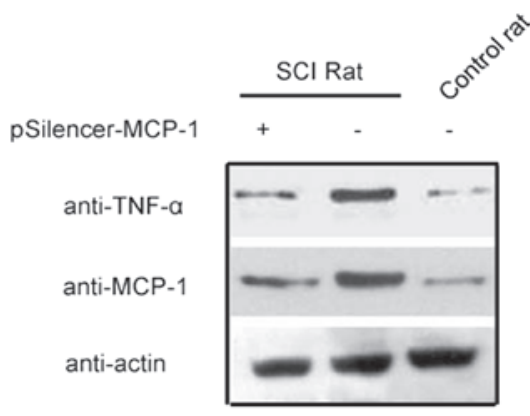

Figure 5. At $24 \mathrm{~h}$ after secondary spinal cord injury (SCI), TNF- $\alpha$ protein expression was increased compared with the sham-surgery control group. TNF- $\alpha$ expression was reduced, but remained higher than that in the control group, following the inhibition of MCP-1 expression. MCP-1, monocyte chemoattractant peptide-1.

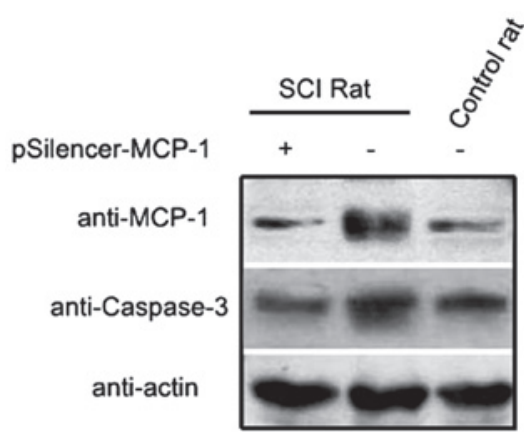

Figure 6. Inhibition of MCP-1 expression affects the expression of caspase-3, which attenuates apoptosis, thus protecting the nerve cells after the emergence of spinal cord injury (SCI). MCP-1, monocyte chemoattractant peptide-1.

In the present study, following SCI, caspase-3 expression levels decreased compared with the control group after the inhibition of MCP-1 expression (Fig. 6). Immunohistochemistry revealed that injury to neuronal cells and astrocytes decreased, suggesting that a reduction in MCP-1 expression inhibits the 
aggravation of apoptosis, thus protecting nerve cells during the secondary SCI process.

In conclusion, our results suggest that inhibiting inflammation alleviates nerve cell injury caused by apoptosis and offers an important approach to the future treatment of secondary SCI. The use of RNAi to inhibit MCP-1 expression, alleviate spinal cord injury and protect nerve cells offers a feasible treatment strategy for patients with this condition. Further research is required to investigate the pathogenesis of secondary SCI and to explore the clinical use of RNAi.

\section{Acknowledgements}

This study was supported by the Young Science Fund Projects of National Natural Science Fund (no. 30801155). We appreciate the valuable advise from other members of our laboratories.

\section{References}

1. Fehlings MG and Perrin RG: The role and timing of early decompression for cervical spinal cord injury: update with a review of recent clinical evidence. Injury 36 (Suppl 2): B13-B26, 2005.

2. Barinaga M: New view of spinal cord injury. Science 274: 1466 1996.

3. Li GL, Farooque M and Olsson Y: Changes of Fas and Fas ligand immunoreactivity after compression trauma to rat spinal cord Acta Neuropathol 100: 75-81, 2000.

4. Emery E, Aldana P, Bunge MB, et al: Apoptosis after traumatic human spinal cord injury. J Neurosurg 89: 911-920, 1998.

5. Whalley K, O'Neill P and Ferretti P: Changes in response to spinal cord injury with development: vascularization, hemorrhage and apoptosis. Neuroscience 137: 821-832, 2006.

6. McTigue DM, Tani M, Krivacic K, et al: Selective chemokine mRNA accumulation in the rat spinal cord after contusion injury. J Neurosci Res 53: 368-376, 1998.
7. Pan JZ, Ni L, Sodhi A, Aguanno A, Young W and Hart RP Cytokine activity contributes to induction of inflammatory cytokine mRNAs in spinal cord following contusion. J Neurosci Res 68: 315-322, 2002.

8. Stammers AT, Liu J, Kwon BK: Expression of inflammatory cytokines following acute spinal cord injury in a rodent model. J Neurosci Res 90: 782-790, 2012.

9. Gourmala NG, Buttini M, Limonta S, Sauter A and Boddeke HW: Differential and time-dependent expression of monocyte chemoattractant protein-1 mRNA by astrocytes and macrophages in rat brain: effects of ischemia and peripheral lipopolysaccharide administration. J Neuroimmunol 74: 35-44, 1997.

10. Miyagishi R, Kikuchi S, Takayama C, Inoue $\mathrm{Y}$ and Tashiro K: Identification of cell types producing RANTES, MIP-1 alpha and MIP-1 beta in rat experimental autoimmune encephalomyelitis by in situ hybridization. J Neuroimmunol 77: 17-26, 1997.

11. Glabinski AR, Balasingam V, Tani M, et al: Chemokine monocyte chemoattractant protein-1 is expressed by astrocytes after mechanical injury to the brain. J Immunol 156: 4363-4368, 1996.

12. Bartholdi D and Schwab ME: Expression of pro-inflammatory cytokine and chemokine mRNA upon experimental spinal cord injury in mouse: an in situ hybridization study. Eur J Neurosci 9: 1422-1438, 1997.

13. Lee SC, Liu W, Dickson DW, Brosnan CF and Berman JW: Cytokine production by human fetal microglia and astrocytes. Differential induction by lipopolysaccharide and IL-1 beta. J Immunol 150: 2659-2667, 1993.

14. Thompson WL and Van Eldik LJ: Inflammatory cytokines stimulate the chemokines CCL2/MCP-1 and CCL7/MCP-3 through NFkB and MAPK dependent pathways in rat astrocytes [corrected]. Brain Res 1287: 47-57, 2009

15. Majumder S, Zhou LZ and Ransohoff RM: Transcriptional regulation of chemokine gene expression in astrocytes. J Neurosci Res 45: 758-769, 1996.

16. Lee YL, Shih K, Bao P, Ghirnikar RS and Eng LF: Cytokine chemokine expression in contused rat spinal cord. Neurochem Int 36: 417-425, 2000.

17. Wang J, Zheng Q, Zhao M and Guo X: Neurocyte apoptosis and expressions of caspase-3 and Fas after spinal cord injury and their implication in rats. J Huazhong Univ Sci Technolog Med Sci 26: 709-712, 2006.

18. Adjan VV, Hauser KF, Bakalkin G, et al: Caspase-3 activity is reduced after spinal cord injury in mice lacking dynorphin: differential effects on glia and neurons. Neuroscience 148: 724-736, 2007. 